\title{
SARS-CoV-2 antibody seroprevalence in NHS healthcare workers in a large double-sited UK hospital
}

\author{
Authors: Gie Ken-Dror, ${ }^{A}$ Charles Wade, ${ }^{B}$ Shyam S Sharma, ${ }^{C}$ Melanie Irvin-Sellers, ${ }^{D}$ Jonathan Robin, ${ }^{\mathrm{E}}$ \\ David Fluck, ${ }^{F}$ Paul Bentley ${ }^{G}$ and Pankaj Sharma ${ }^{H}$
}

\begin{abstract}
We determined the seroprevalence of SARS-CoV-2 antibodies in NHS healthcare workers (HCWs) in a cross-sectional study from a large general hospital located in a double-sited rural and semi-rural area. The sample size of 3,119 HCWs (mean age $43 \pm 13)$ consisted of $75.2 \%$ women, $61.1 \%$ White individuals and predominantly (62.4\%) asymptomatic individuals. Seroprevalence of SARS-CoV-2 antibodies was $19.7 \%$. Determinants of seropositivity were preceding symptomatic infection and non-White ethnicity. Regardless of staff role or sex, multivariate regression analysis revealed that non-White HCWs were three times (odds ratio [OR] 3.12, 95\% confidence interval [CI] 2.53-3.86, $\mathrm{P}<0.001$ ) more likely to have antibodies than White staff, and seven times (OR 7.10, 95\% CI 5.72-8.87, $\mathrm{P}<0.001)$ more likely if there was a history of preceding symptoms. We report relatively high rates of seropositivity in all NHS healthcare workers. Non-White symptomatic HCWs were significantly more likely to be seropositive than their colleagues, independent of age, sex or staff role.
\end{abstract}

KEYWORDS: COVID-19, SARS-CoV-2, coronavirus, seropositive, antibody, NHS staff, healthcare workers

DOI: $10.7861 /$ clinmed.2020-1096

\section{Introduction}

Severe acute respiratory syndrome coronavirus 2 (SARS-CoV-2), the virus responsible for the coronavirus disease 2019 (COVID-19)

Authors: Apostdoctoral research fellow, Institute of Cardiovascular Research, Royal Holloway University of London, Egham UK;

${ }^{B} S p R$ neurology, Imperial College NHS Healthcare Trust, London; Cmedical student, Edinburgh Medical School, University of

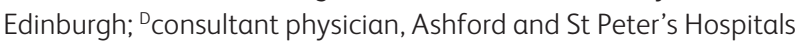
NHS Foundation Trust, Surrey, UK; ${ }^{\mathrm{E}}$ Consultant physician, Ashford and St Peter's Hospitals NHS Foundation Trust, Surrey, UK; Fmedical director, Ashford and St Peter's Hospitals NHS Foundation Trust, Surrey, UK; ${ }^{\mathrm{G}}$ senior lecturer, Division of Clinical Neuroscience, Imperial College London, London, UK; ${ }^{H}$ professor of neurology and director, Institute of Cardiovascular Research, Royal Holloway University of London, and consultant neurologist, Imperial College Healthcare NHS Trust, London, UK pandemic, stimulates an antibody response in both symptomatic and asymptomatic infection. ${ }^{1,2}$ Healthcare workers (HCWs) were shown to be at increased risk of infection in previous coronavirus outbreaks, ${ }^{3}$ making up $21.3 \%$ of total severe acute respiratory syndrome (SARS) infections and $18.9 \%$ of Middle East respiratory syndrome (MERS) infections. ${ }^{4}$ Available data suggest they remain at high risk of COVID-19, ${ }^{5}$ and more likely to show a positive COVID-19 antigen test result compared to the general population. Identifying seropositive HCWs is important at an individual level (though it is not yet known whether antibodies correlate with immunity or how long titres are maintained) but also on a wider scale. In determining staff seroprevalence patterns, we not only gain a surrogate marker for community transmission in the populations for which they cater, but also contribute to our understanding of hospital and nosocomial spread and the effectiveness of our infection control policies. ${ }^{6}$ When comparing SARS-CoV-2 seropositivity of HCWs across large areas, differences in personal factors like demographic or health status can be complicated by local organisational differences, for example in hospital infection rates or personal protective equipment (PPE) policies.

We sought to quantify SARS-CoV-2 seroprevalence in HCWs and therefore provide a better understanding of whether current protective measures are adequate for reducing pressures on healthcare organisations or if additional and more individualised measures are needed to prevent future staff illness, thus enhancing staff safety, preventing outbreaks and limiting staff shortages, regardless of the geographical location of the health organisation.? We report the seroprevalence of SARS-CoV-2 antibodies among $\mathrm{HCWs}$ and investigate if specific subgroups are more likely to seroconvert.

\section{Methods}

All adult staff ( $\geq 18$ years old) from Ashford and St Peters Hospital (ASPH), Surrey, UK were invited for SARS-CoV-2 IgG antibody testing using Abbott SARS-CoV-2 IgG (Abbott Laboratories, Chicago, USA) kit between October and November 2020. The sensitivity of this test is $82.5 \%$ (95\% CI 75.3-88.4) and the specificity $99.5 \%$ (95\% CI 98.7-99.9). ${ }^{8,9}$ The hospital is a busy dual-site district general hospital located within and beyond the greater London area, thus encompassing both urban and semi-rural settings. Self-reported data on exposure history, typical symptomatology, comorbidities, treatment, complications and outcome were recorded. Ethical approval was obtained from ASPH Ethics Committee. 
Table 1. Patient demographics, comorbidities and symptoms, by antibody status of SARS-CoV-2

\begin{tabular}{|c|c|c|c|c|}
\hline Characteristic & All sample $(n=3119)$ & Positive antibody $(n=613)$ & Negative antibody $(n=2,506)$ & P-value \\
\hline \multicolumn{5}{|l|}{ Age, years $n(\%)$} \\
\hline$\leq 30$ & $669(21.4 \%)$ & $155(25.3 \%)$ & $514(20.5 \%)$ & \\
\hline $31-40$ & $671(21.5 \%)$ & $122(19.9 \%)$ & $549(21.9 \%)$ & \\
\hline $41-50$ & $812(26.0 \%)$ & $183(29.9 \%)$ & $629(25.1 \%)$ & \\
\hline $51-60$ & $702(22.5 \%)$ & $118(19.2 \%)$ & $584(23.3 \%)$ & \\
\hline$\geq 61$ & $265(8.5 \%)$ & $35(5.7 \%)$ & $230(9.2 \%)$ & $<0.001$ \\
\hline \multicolumn{5}{|l|}{ Sex } \\
\hline Male, n (\%) & $775(24.8 \%)$ & $178(29.0 \%)$ & $597(23.8 \%)$ & \\
\hline Female, n (\%) & $2,344(75.2 \%)$ & $435(71.0 \%)$ & $1,909(76.2 \%)$ & 0.009 \\
\hline \multicolumn{5}{|l|}{ Ethnicity $\left(n=2,953^{*}\right)$ : } \\
\hline White, n (\%) & $1,804(61.1 \%)$ & $225(39.1 \%)$ & $1,579(66.4 \%)$ & \\
\hline Non-White, n (\%) & $1,149(38.9 \%)$ & $350(60.9 \%)$ & $799(33.6 \%)$ & $<0.001$ \\
\hline \multicolumn{5}{|l|}{ Symptoms $\left(n=2,790^{+}\right)$: } \\
\hline No, $\mathrm{n}(\%)$ & $1,740(62.4 \%)$ & $151(26.4 \%)$ & $1,589(71.7 \%)$ & \\
\hline Yes, n (\%) & $1,050(37.6 \%)$ & $422(73.6 \%)$ & $628(28.3 \%)$ & $<0.001$ \\
\hline Fever, $\mathrm{n}(\%)$ & $154(4.9 \%)$ & $98(16.0 \%)$ & $56(2.2 \%)$ & $<0.001$ \\
\hline Cough, $\mathrm{n}(\%)$ & $154(4.9 \%)$ & $84(13.7 \%)$ & $70(2.8 \%)$ & $<0.001$ \\
\hline Shortness breath, n (\%) & $47(1.5 \%)$ & $27(4.4 \%)$ & $20(0.8 \%)$ & $<0.001$ \\
\hline Loss smell/taste, n (\%) & $62(2.0 \%)$ & $54(8.8 \%)$ & $8(0.3 \%)$ & $<0.001$ \\
\hline Headache, $n(\%)$ & $92(2.9 \%)$ & $47(7.7 \%)$ & $45(1.8 \%)$ & $<0.001$ \\
\hline Fatigue, $\mathrm{n}(\%)$ & $95(3.0 \%)$ & $58(9.5 \%)$ & $37(1.5 \%)$ & $<0.001$ \\
\hline Sore throat, n (\%) & $73(2.3 \%)$ & $31(5.1 \%)$ & $42(1.7 \%)$ & $<0.001$ \\
\hline Myalgia, n (\%) & $104(3.3 \%)$ & $68(11.1 \%)$ & $36(1.4 \%)$ & $<0.001$ \\
\hline Diarrhoea, $\mathrm{n}(\%)$ & $20(0.6 \%)$ & $9(1.5 \%)$ & $11(0.4 \%)$ & 0.009 \\
\hline Nausea/vomiting, n (\%) & $19(0.6 \%)$ & $14(2.3 \%)$ & $5(0.2 \%)$ & $<0.001$ \\
\hline Runny nose, n (\%) & $17(0.5 \%)$ & $12(2.0 \%)$ & $5(0.2 \%)$ & $<0.001$ \\
\hline Dizziness/vertigo, n (\%) & $5(0.2 \%)$ & $2(0.3 \%)$ & $3(0.1 \%)$ & 0.26 \\
\hline Back pain, $\mathrm{n}(\%)$ & $7(0.2 \%)$ & $5(0.8 \%)$ & $2(0.1 \%)$ & 0.004 \\
\hline Loss appetite, n (\%) & $10(0.3 \%)$ & $10(1.6 \%)$ & 0 & - \\
\hline Chest pain/tightness, n ( \%) & $13(0.4 \%)$ & $7(1.1 \%)$ & $6(0.2 \%)$ & 0.006 \\
\hline \multicolumn{5}{|l|}{ Staff group } \\
\hline Admin and estates & $833(26.7 \%)$ & $128(20.9 \%)$ & $705(28.1 \%)$ & \\
\hline Clinical support & $615(19.7 \%)$ & $135(22.0 \%)$ & $480(19.2 \%)$ & \\
\hline Medical & $459(14.7 \%)$ & $90(14.7 \%)$ & $369(14.7 \%)$ & \\
\hline Nursing and midwifery & $951(30.5 \%)$ & $226(36.9 \%)$ & $725(28.9 \%)$ & \\
\hline Other clinical registered & $261(8.4 \%)$ & $34(5.5 \%)$ & $227(9.1 \%)$ & $<0.001$ \\
\hline
\end{tabular}

\section{Statistical analysis}

Descriptive statistics were summarised using mean with standard deviation (SD) or median with interquartile range (IQR) for continuous variables and proportion for categorical variables. The chi-square or Fisher's exact test was used for single factor analysis of categorical variables. Independent t-test or Mann-Whitney $U$ test was used for single factor analysis of continuous variables. Univariate and multivariate logistic regression were used to estimate the associations of risk factors with SARS-CoV-2 seropositivity status. Regression coefficients and odds ratios (OR) were calculated for independent risk factors. Models were evaluated using Akaike's information criterion (AIC) and the likelihood ratio chi-square test. ${ }^{10}$ Receiver operating characteristic (ROC) curve and the Youden index (YI) measurements corresponding to the maximum YI value were considered the best diagnostic values. ${ }^{10,11}$ For individual tests of association, rather than applying a correction for multiple testing at global significance level, statistical significance was defined as 0.01 . $^{7}$ Analyses and graphics were performed and produced using $\mathrm{R}$ version 4.0.0.

\section{Results}

Of the 4,000 total staff members invited for testing, 3,119 (78\%) responded and were available for analysis and provided demographic data and data on staff role, prior symptoms and antibody test results. The sample (mean age: $43 \pm 13$ ) consisted of 2,344 (75.2\%) females, $1,804(61.1 \%)$ White individuals and 1,740 (62.4\%) asymptomatic individuals. HCWs were divided into 951 nursing and midwifery (30.5\%), 833 administrative and estates (26.7\%), 615 clinical support (19.7\%), 459 medical (14.7\%) and 261 other clinical registered persons (8.4\%). Of 3,119 HCWs, $613(19.7 \%)$ returned positive antibody tests. Patient demographics, comorbidities and symptoms by antibody status of SARS-CoV-2 are shown in Table 1.

$23 \%(n=178)$ of male staff were seropositive, versus $18.6 \%$ ( $n=435$ ) of female staff, meaning that $29 \%$ of the positive antibody tests were seen in males despite their making up only $24.8 \%$ of the sample ( $O R=1.31,95 \%$ CI: $1.07-1.59, P=0.008$ ). More than $30 \%(n=350)$ of non-White staff were seropositive versus $12.5 \%(n=225)$ of White staff, meaning that $60.9 \%$ of 


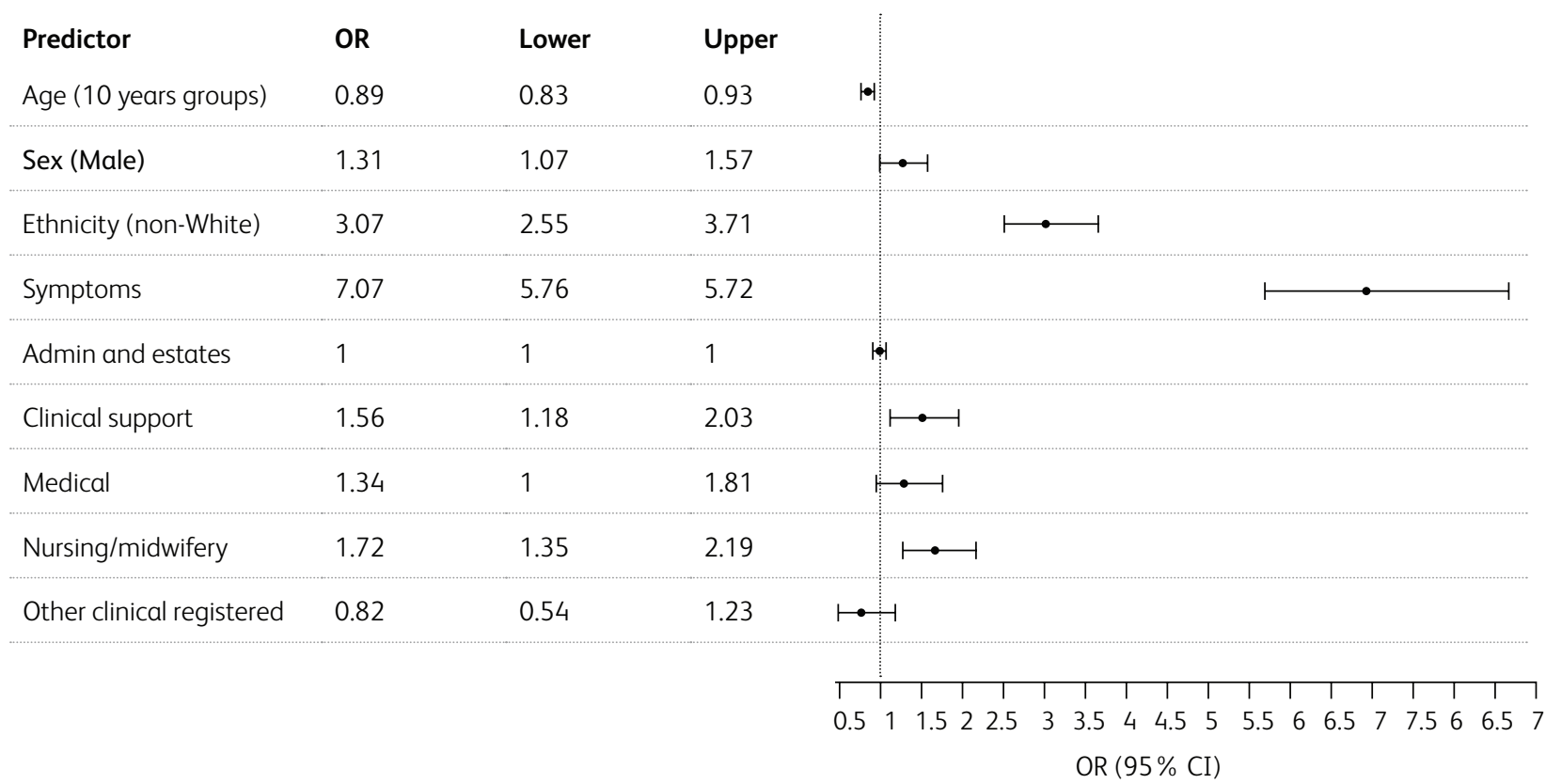

Fig 1. Forest plot showing the odds ratio of positive antibody as outcome using logistic regression analysis. OR, odds ratio.

positive antibody tests were seen in non-White staff members (OR 3.07 , 95\% CI 2.55-3.71, $\mathrm{P}<0.001$ ), despite making up only $38.9 \%$ of the sample (Table 1).

Seropositivity was most seen $(22.5 \%)$ in the $41-50$-year age group and least observed (13.2\%) in those over 60 years of age $(\delta 9.3 \%, 95 \%$ CI $4.1-14.6 \%, P=0.001)$, although there were few data on the latter group. Staff with direct clinical exposure were more likely to have positive antibody tests. Nursing and midwifery staff were most likely to be seropositive ( $23.8 \%$ of those tested), representing $36.9 \%$ of total tests, followed by clinical support staff (22.0\%) and medical staff (19.6\%) and then administrative and estates staff $(15.4 \%)$.

Almost 38\% ( $n=1,050)$ staff members had a symptomatic illness prior to antibody testing. Presence of symptoms was a statistically significant $(\mathrm{P}<0.001)$ predictor of antibody positivity. More than $40 \%(n=422)$ of those with a history of symptoms suggestive of SARS-CoV-2 were found to be antibody positive, compared with just $8.7 \%(n=151)$ who were asymptomatic throughout. $25 \%$ of positive antibody results were returned by asymptomatic people, indicating that a significant percentage of infections are mild or asymptomatic. Though presence of all symptoms (except dizziness/vertigo) correlated significantly with antibody positivity, the most associated were myalgia $(65.4 \%)$, fever $(63.6 \%)$ and cough (54.5\%). $87 \%$ of those who reported anosmia were antibody-positive (Table 1), although the size of the available dataset here was small.

To evaluate the association of ethnicity with the presence of positive SARS-CoV-2 antibodies, a logistic regression was performed for the following variables: age, sex, symptoms and staff group. Univariate analysis showed that age, sex, ethnicity, symptoms and staff group were associated with positive SARS-CoV-2 antibodies (Fig 1). The OR for positive SARS-CoV-2 antibodies presence was 3.07 (95\% CI 2.55-3.71, $\mathrm{P}<0.001$ ) for non-White ethnicity.

Multivariate logistic regression analysis modelling five risk factors (age, sex, ethnicity, symptoms and staff group) showed that sex, ethnicity, and reported symptoms were each independently associated with positive SARS-CoV-2 antibodies (Table 2).

Although age and staff group were associated with positive SARS CoV-2 antibodies in the univariate analysis, they were not found to be independently associated with positive SARS-CoV-2 antibodies in this multivariate analysis. The ROC curve showed that age and staff group had no statistically significant effect on the presence of positive SARS-CoV-2 antibodies. Multivariate adjusted OR for non-White ethnicity was 3.01 (95\% CI 2.42-3.76, P<0.001).

\section{Discussion}

In this large cross-sectional study of symptomatic and asymptomatic healthcare workers, seroprevalence of SARS-CoV-2 antibodies was $19.7 \%$, which is considerably greater than the $4.4 \%$ seroprevalence in the general population in the South East region determined by Public Health England.12 The 1,149 non-White staff members represented $38.9 \%$ of all participants, which is a higher proportion than in the population of Surrey as a whole $(10 \%))^{13}$ This may be related to a high level of viral load to which staff are chronically exposed. We show that staff members who report a preceding symptomatic illness were seven times more likely $(\mathrm{OR}=7.10$, $95 \%$ CI 5.72-8.87, P<0.001) to show antibodies than those with no preceding symptoms ( $P<0.001)$. Our estimate relates to asymptomatic development of SARS-CoV-2 antibodies rather than asymptomatic carriage of coronavirus, as it has been reported that not all who have COVID-19 develop detectable antibodies.14

These data add to similar studies that also find elevated infection rates and seroprevalence in HCWs compared with the general population, suggesting a marked occupational risk of exposure to SARS-CoV-2..$^{15}$ That said, seroprevalence rates seem to vary considerably intra- and internationally. Whereas other UK studies have found similar rates of seroprevalence in HCWs (Gateshead 19.4\%, Birmingham 24.4\%) ${ }^{16,17}$, the prevalence of SARS-CoV-2 antibody seropositivity was lower in 
Table 2. Multivariate logistic regression analysis of SARS-CoV-2 antibodies status

\begin{tabular}{|c|c|c|c|}
\hline Predictor & Model 1 & Model 2 & Model 3 \\
\hline $\begin{array}{l}\text { Ethnicity (non-White), } \\
\text { OR (CI), p-value }\end{array}$ & 3.07 (2.55-3.71), 0.001 & $3.12(2.53-3.86),<0.001$ & $3.01(2.42-3.76),<0.001$ \\
\hline Symptoms (yes), OR (CI), p-value & & $7.10(5.72-8.87),<0.001$ & 7.19 (5.77-9.00), <0.001 \\
\hline Sex (male), OR (CI), p-value & & & 1.33 (1.02-1.72), 0.032 \\
\hline $\begin{array}{l}\text { Staff group, OR (CI), p-value } \\
\text { Admin and estates } \\
\text { Clinical support } \\
\text { Medical } \\
\text { Nursing/midwifery } \\
\text { Other clinical registered }\end{array}$ & & & $\begin{array}{l}\text { Reference group } \\
1.09 \text { (0.79-1.50), } 0.61 \\
0.73 \text { (0.51-1.06), } 0.10 \\
1.11 \text { (0.83-1.50), } 0.47 \\
0.75 \text { (0.47-1.19), } 0.24\end{array}$ \\
\hline AIC & $2,774.1$ & $2,196.3$ & 2,196.6 \\
\hline Sensitivity/specificity & $0.61 / 0.66$ & $0.73 / 0.72$ & $0.73 / 0.72$ \\
\hline $\mathrm{AUC}(\mathrm{CI})$ & $0.64(0.61-0.66)$ & $0.78(0.76-0.80)$ & $0.79(0.77-0.81)$ \\
\hline
\end{tabular}

New York $(13.7 \%)^{18}$, Barcelona $(9.3 \%)$ and the Capital Region of Denmark (4.04\%). ${ }^{19,20}$ Indeed, a study of SARS-CoV-2 antibodies in Kerala, India found that even 5 months after the report of the first case of COVID-19 there was no prevalence of the SARS-CoV-2 antibody in $\mathrm{HCWs} .{ }^{21} \mathrm{~A}$ possible consequence of $\mathrm{HCW}$ infection, $\mathrm{Xu}$ et $a{ }^{22}$ found seropositivity rates of $3.2 \%$ in relatives of those who worked in hospitals (versus $0.6 \%$ in other community residents), ${ }^{22} \mathrm{a}$ finding supported by work in Belgium where household contacts of seropositive HCWs were 3.15 times as likely (95\% CI 2.33-4.25) to show antibody positivity than those without this exposure. ${ }^{23}$ There are likely numerous reasons for these reported wide variations in HCW seropositivity, including (but not limited to) antibody assays used, time points of testing (eg different phases of the pandemic), distributions of patients (eg into 'COVID-19 hospitals' and 'nonCOVID-19 hospitals'), as well as differences in local guidance on, and availability of, personal protective equipment. It should be noted that despite working in a hospital located in an affluent, socially homogeneous and relatively healthy region ${ }^{7}$ where seropositivity levels in the general population are low, ${ }^{12}$ our HCWs exhibited similar seroprevalence levels to other HCWs working in more deprived areas where the general population was more likely to be seropositive. ${ }^{12}$

More than $30 \%$ of all non-White staff were found to be antibody positive and we demonstrate that non-White ethnicity confers a significantly increased risk of seropositivity. Multivariate regression analysis revealed that non-White staff members were three times more likely to have antibodies than White staff members. Importantly, this significance was maintained regardless of staff role or gender $(P<0.001)$. This result is in line with work by others showing that ethnic minority groups have been disproportionately affected by COVID-19. Shields et a ${ }^{17}$ demonstrated that staff of BAME (Black, Asian and minority ethnic) ethnicity in Birmingham UK were nearly twice as likely to be seropositive (adjusted OR 1.92, $95 \%$ CI $1.14-3.23, P=0.01$ ) than individuals of White ethnicity. ${ }^{17}$ In this study a (non-significant) link was made between ethnicity and living in significantly more deprived areas. ${ }^{17}$ While postcodes of staff in our study were not collected, it should be noted that the gross regional domestic product per capita and healthy life expectancy of Surrey are both within the UK top deciles, perhaps making living conditions less relevant here. ${ }^{24,25}$ An analysis by the Washington Post reports that those counties with Black majorities have three times the rate of COVID-19 cases compared with counties where White residents are in the majority. ${ }^{26}$ Much work has been done highlighting potential racial, economic and other inequalities that lead to this. By extracting data from a single UK centre, our study suggests that an increased risk of seropositivity in non-White staff arises not just from a greater risk of exposure to the SARS-CoV-2 virus but also a greater chance of seroconversion once exposed.

We show no statistically significant difference in seropositivity rates between different types of patient-facing staff groups. Early in the pandemic it had been assumed that HCWs in certain specialties (eg anaesthetics and intensive care) would be at increased risk of infection due to a perceived increased exposure to COVID-19 patients and the performance of high-risk procedures. ${ }^{16,20}$ Our data, and other data ${ }^{19}$ including a recent large analysis of three separate studies from Oxford, Leicester and Birmingham ${ }^{17}$ with more than 20,000 healthcare staff, suggest that this is not necessarily the case. In fact, Cook et a ${ }^{27}$ indicate that those working in anaesthesia and intensive care actually had less than half the risk of infection than physicians dealing with COVID-19 patients on the wards This could be due to consistent use of similar personal protective equipment (PPE) across staff, improved risk-mitigation by those in 'higher risk' groups and working in well ventilated environments. ${ }^{27}$

There are limitations to our cross-sectional study. Data were not available to determine the representativeness of our sampling in terms of overall staff at the hospital or on the possible confounding risk factors for staff with underlying health conditions, large body mass index (BMI) or indices of deprivation in participants' postcodes. By failing to capture more recent infections leading to seroconversion, this may underestimate the true seroprevalence, although our study will likely have captured the peak of the pandemic. A current consideration with regards to seroprevalence of antibodies in COVID-19 is the longevity of seropositivity, for which we have no data. Further, without parallel PCR testing alongside symptom tracking, we cannot be certain whether seronegative individuals reporting COVID-19 symptoms 
either had symptoms secondary to an unrelated infection or simply did not develop detectable SARS-CoV-2 antibodies despite suffering with the virus. Lack of specific data regarding symptoms limited our ability to granulate analysis of seropositivity with individual symptoms. Further studies are necessary to understand the increased risk of seropositivity observed within individuals of non-White ethnicity and understand if this is associated with the observed increased risk of mortality. Other important questions relating to seropositivity, such as its impact on health, outcome and hospitalisation as well as likelihood of protection against future infection or virus transmissibility, are not able to be addressed by our study.

\section{Conclusions}

We document high seroprevalence of SARS-CoV-2 antibodies in healthcare workers. Independent of age, sex or specific staff role, non-White staff have significantly increased seroprevalence, suggesting a differential risk.

\section{References}

1 Assadiasl S, Fatahi Y, Zavvar M, Nicknam MH. COVID-19: Significance of antibodies. Hum Antibodies 2020;28:287-97.

2 Paces J, Strizova Z, Smrz D, Cerny J. COVID-19 and the immune system. Physiol Res 2020;69:379-88.

3 Chowell G, Abdirizak F, Lee $S$ et al. Transmission characteristics of MERS and SARS in the healthcare setting: a comparative study. BMC Med 2015;13:210.

4 Xiao J, Fang M, Chen Q, He B. SARS, MERS and COVID-19 among healthcare workers: A narrative review. J Infect Public Health 2020 13:843-8.

5 Nguyen LH, Drew DA, Graham MS et al. Risk of COVID-19 among front-line health-care workers and the general community: a prospective cohort study. Lancet Public Health 2020;5:e475-83.

6 Verbeek JH, Rajamaki B, Ijaz S et al. Personal protective equipment for preventing highly infectious diseases due to exposure to contaminated body fluids in healthcare staff. Cochrane Database Syst Rev 2020;4:CD011621.

7 Ken-Dror G, Wade C, Sharma S et al. COVID-19 outcomes in UK centre within highest health and wealth band: a prospective cohort study. BMJ Open 2020;10:e042090.

8 Choy KW. Specificity and cross-reactivity of a test for anti-SARSCoV-2 antibodies. Lancet Infect Dis 2020, in press (doi: 10.1016/ S1473-3099(20)30787-8)

9 Iversen K, Bundgaard H, Harritshoj L, Ullum H. Specificity and crossreactivity of a test for anti-SARS-CoV-2 antibodies - Authors' reply. Lancet Infect Dis 2020, in press (doi: 10.1016/ S1473-3099(20) 30849-5).

10 Zhou X-H, McClish DK, Obuchowski NA. Statistical methods in diagnostic medicine. Wiley, 2011.

11 Faraggi D, Reiser B. Estimation of the area under the ROC curve. Stat Med 2002;21:3093-106.
12 Public Health England. Weekly coronavirus disease 2019 (COVID 19) surveillance report. www.gov.uk/government/publications/ national-covid-19-surveillance-reports.

13 Surrey Heath. Census 2011. www.surreyheath.gov.uk/business/ economic-development/census-and-demographic-information/ census-2011.

14 Solbach W, Schiffner ], Backhaus I et al. Antibody profiling of COVID-19 patients in an urban low-incidence region in northern Germany. Front Public Health 2020;8:570543.

15 Lai CC, Wang JH, Hsueh PR. Population-based seroprevalence surveys of anti-SARS-CoV-2 antibody: An up-to-date review. Int J Infect Dis 2020;101:314-22.

16 Razvi S, Oliver R, Moore J, Beeby A. Exposure of hospital healthcare workers to the novel coronavirus (SARS-CoV-2). Clin Med 2020; 20:e238-e40.

17 Shields A, Faustini SE, Perez-Toledo M et al. SARS-CoV-2 seroprev alence and asymptomatic viral carriage in healthcare workers: a cross-sectional study. Thorax 2020;75:1089-94.

18 Moscola J, Sembajwe G, Jarrett M et al. Prevalence of SARS-CoV-2 antibodies in health care personnel in the New York City area. JAMA 2020;324:893-5.

19 Garcia-Basteiro AL, Moncunill G, Tortajada M et al. Seroprevalence of antibodies against SARS-CoV-2 among health care workers in a large Spanish reference hospital. Nat Commun 2020;11:3500.

20 Iversen K, Bundgaard H, Hasselbalch RB et al. Risk of COVID-19 in health-care workers in Denmark: an observational cohort study. Lancet Infect Dis 2020;20:1401-8.

21 Kumar A, Sathyapalan D, Ramachandran A et al. SARS-CoV-2 antibodies in healthcare workers in a large university hospital, Kerala, India. Clin Microbiol Infect 2021;27:481-3.

22 Xu X, Sun J, Nie S et al. Seroprevalence of immunoglobulin $\mathrm{M}$ and $\mathrm{G}$ antibodies against SARS-CoV-2 in China. Nat Med 2020;26:1193-5.

23 Steensels D, Oris E, Coninx L et al. Hospital-Wide SARS-CoV-2 Antibody Screening in 3056 Staff in a Tertiary Center in Belgium. JAMA 2020;324:195-7.

24 Surrey Public Health. Joint strategic needs assessment: Long term conditions. www.surreyi.gov.uk/jsna/long-term-conditions/.

25 Surrey Public Health. Joint strategic needs assessment: Economy, employment, deprivation. www.surreyi.gov.uk/jsna/economyemployment-and-deprivation/.

26 Khunti K, Singh AK, Pareek M, Hanif W. Is ethnicity linked to incidence or outcomes of Covid-19? BMJ 2020;369:m1548.

27 Cook TM, Lennane S. Occupational COVID-19 risk for anaesthesia and intensive care staff - low-risk specialties in a high-risk setting. Anaesthesia 2020;76:295-300.

Address for correspondence: Professor Pankaj Sharma, Institute of Cardiovascular Research, Royal Holloway University of London (ICR2UL), TW20 0EX, UK. Email: pankaj.sharma@rhul.ac.uk 